\title{
PERFECCIONISMO, BIENESTAR PSICOLÓGICO Y DISPOSICIÓN A FLUIR EN ENFERMERAS d
}

\author{
Diorelis Josefina Colmenares Martínez ${ }^{\mathbb{D}}$, \& Leonardo Andrés Aguilar \\ Durán ${ }^{1}$ [C
}

Universidad Central de Venezuela, Caracas, Venezuela.

\begin{abstract}
RESUMEN
El objetivo de este trabajo fue evaluar la relación entre el perfeccionismo, el bienestar psicológico y la disposición a fluir en enfermeras. Se trató de un estudio cuantitativo con alcance descriptivo-correlacional. Participaron 150 enfermeras $\left(M_{\text {edad }}=41.95, D E=11.24\right)$ que laboraban en instituciones de salud públicas y privadas de Caracas. Las variables se midieron con instrumentos de autoinforme. En el análisis de los datos se empleó estadística descriptiva y se calcularon correlaciones bivariadas. Se encontraron niveles moderados de perfeccionismo, junto a altos niveles de bienestar y flujo. El perfeccionismo se relacionó positivamente con el bienestar y con la disposición a fluir $(p<.01)$. Los resultados destacan el ajuste psicológico del personal de enfermería y cuestionan la visión negativa imperante sobre el perfeccionismo.
\end{abstract}

\section{Palabras Claves}

perfeccionismo, bienestar psicológico, disposición a fluir, enfermeras venezolanas

\begin{abstract}
The objective of this research was to evaluate the relationship between perfectionism, psychological wellbeing and dispositional flow in nurses. A quantitative study with descriptive-correlational scope was designed. The sample consisted of 150 nurses (mean age $=41.95, S D=11.24$ ) who were working in public and private health institutions of Caracas. The variables were measured with self-report instruments. Descriptive statistics and bivariate correlations were used in the analysis of the data. Moderate levels of perfectionism were found, along with high levels of well-being and flow. Perfectionism was positively related to well-being and dispositional flow $(p<.01)$. The results highlight the psychological adjustment of the nursing staff and contradict the prevailing negative view on perfectionism.
\end{abstract}

\section{Keywords}

perfectionism, psychological well-being, dispositional flow, Venezuelan nurses

\footnotetext{
${ }^{1}$ Correspondence about this article should be addressed to Leonardo Andrés Aguilar Durán: psileonardo@gmail.com
} 


\section{PERFECTIONISM, PSYCHOLOGICAL WELL-BEING AND DISPOSITIONAL FLOW IN NURSES}

\section{Introducción}

En la que podría ser una de las definiciones más difundidas del perfeccionismo, Frost et al. (1990) lo conceptualizan como "la tendencia a establecer altos estándares de desempeño que se acompaña de una evaluación crítica de ese desempeño y una preocupación excesiva por cometer errores" (p. 450). El modelo venezolano abreviado del constructo (Prieto \& Vento, 2012) diferencia tres dimensiones: (1) preocupación por los errores, es decir, la tendencia del sujeto a interpretar las equivocaciones como fracasos y a creer que los demás le perderán el respeto por ello; (2) organización, se refiere a la importancia que se otorga al orden y la preferencia por el mismo; y (3) expectativas paternas, o la percepción del individuo de que sus padres tienen expectativas elevadas sobre él.

Hay en la literatura científica un debate acerca de si el perfeccionismo es un rasgo positivo o negativo, en términos prosaicos: sobre si es bueno o malo ser alguien perfeccionista. Quienes trabajan amparados en la formulación de Frost et al. (1990) piensan que se trata de un atributo más indeseable que saludable. Afirman esto en razón de que numerosas investigaciones han demostrado la relación del perfeccionismo con estados de ánimo negativos (e.g., culpa, indecisión, vergüenza, ira) y con una variedad de problemas clínicos como estrés, depresión, ansiedad, trastornos del comportamiento alimentario, trastorno obsesivo compulsivo, fobia social, alcoholismo, entre otros. No obstante, de las antedichas, la preocupación por los errores y las expectativas de los padres son las dimensiones del perfeccionismo que en mayor medida se asocian con la psicopatología (Cheng et al., 1999; Franco, 2007; Franco et al., 2010; Frost et al., 1993).

El perfeccionismo es un rasgo de la personalidad que impregna todos los ámbitos de la vida. De allí que durante los últimos veinte años, su estudio se haya ido incrementando exponencialmente en los más diversos contextos, sobre todo en el clínico y el educativo, mientras que ha sido relativamente ignorado en el contexto laboral, donde puede ejercer alguna influencia sobre la salud y el bienestar de los empleados (Ozbilir et al., 2015).

El perfeccionismo es susceptible de observarse en el entorno laboral porque muchas organizaciones esperan que sus empleados minimicen, casi por completo, la ocurrencia de errores como parte del desempeño, para de esa forma cumplir con los 
propósitos de la empresa u organización (Stoeber \& Damian, 2016). En el área laboral de la salud el perfeccionismo parece cobrar mucha relevancia, ya que las instituciones hospitalarias tienden a exigir la perfección a los trabajadores, esto con el fin de evitar que los pacientes sufran consecuencias que puedan perjudicar su salud. Aunque la investigación sobre el perfeccionismo en la vida laboral de las personas todavía es limitada, estudios previos con empleados de diversa índole (Kanten \& Yesiltas, 2015; Ozbilir et al., 2015; Stoeber \& Damian, 2016) y con trabajadores de la salud - como psicólogos clínicos (D’Souza et al., 2011), médicos (Craiovan, 2014) y enfermeras (Chang, 2012, 2017) — indican que se trata de un rasgo que puede incrementar el rendimiento de los empleados, pero que, en exceso, puede repercutir negativamente en la productividad, perturbar la salud y afectar las relaciones con los compañeros de trabajo.

La enfermería es una ocupación que se revela como de particular importancia al examinar el efecto del perfeccionismo en el trabajo. Las profesionales de este ramo de la salud laboran en un entorno que demanda una actuación con elevados estándares de calidad, donde los errores pueden comprometer la seguridad del paciente y el buen resultado de los procedimientos médicos (Chang, 2012; Melrose, 2011). Asimismo, se observa que conductas que involucran minuciosidad, orden, limpieza, responsabilidad y paciencia forman parte del perfil del personal de enfermería (Kozier et al., 2008).

La segunda variable en este estudio es el bienestar psicológico. Para SánchezCánovas (2007), el bienestar se refiere a la sensación de felicidad como experiencia subjetiva. En este sentido, el autor distingue cuatro facetas, tres de las cuales resultan de interés: (1) bienestar subjetivo, hace referencia a la percepción de felicidad en la etapa vital que el individuo está atravesando; (2) bienestar material, está basado en la satisfacción que generan los ingresos económicos y las posesiones materiales cuantificables; y (3) bienestar laboral, se basa en la satisfacción con el trabajo como componente importante de la satisfacción general.

En lo que respecta a las enfermeras, la investigación ha señalado que el bienestar de estas profesionales redunda en la calidad de la asistencia que brindan y, en última instancia, en el bienestar físico y emocional de los propios pacientes atendidos (Newman et al., 2002). Sin embargo, estudios realizados en varios países indican que desempeñarse como enfermera implica una alta propensión a experimentar estrés y desgaste profesional (Fiabane et al., 2013; Mark \& Smith, 2012; Sahraian et al., 2008), además de problemas conexos como ansiedad y depresión (Burke, 2003; Chang, 2012; Chang et al., 2011), al igual que supone un riesgo elevado de sufrir lesiones musculoesqueléticas y de contraer 
infecciones en el ambiente hospitalario (Fronteira \& Ferrinho, 2011). Entre las condiciones laborales adversas a las que deben hacer frente las enfermeras se cuentan: fuertes cargas de trabajo, jornadas extras y en turnos rotativos, baja remuneración, dificultades en la comunicación intralaboral (especialmente con los médicos) y, por supuesto, el frecuente contacto cara a cara con el sufrimiento humano. Todo lo cual señala a la enfermería como una ocupación que, aunque ocupa un lugar central en la prestación de los servicios de salud, genera poco interés en especializarse y registra una alta intención de abandono entre sus practicantes (Aiken et al., 2001; Gardulf et al., 2005). Por si fuese poco, el panorama descrito debe apreciarse en el contexto venezolano, donde el gremio de las enfermeras es uno de los que se mantiene en conflicto con el Gobierno nacional en el marco de la crisis del sistema de salud que sufre el país.

De acuerdo con Seligman (2011), un precursor fundamental del bienestar psicológico es la disposición a fluir o simplemente flujo, la tercera variable de interés en esta investigación. Se trata de un estado subjetivo que las personas experimentan cuando están involucradas por completo en una actividad, hasta el punto de olvidarse del tiempo, la fatiga y de todo lo demás, excepto de la actividad misma (Csikszentmihalyi, 2009). En el ámbito laboral, indican Csikszentmihalyi y Csikszentmihalyi (1998) que un trabajador entra en estado de flujo cuando tiene la sensación de que sus habilidades son adecuadas para enfrentarse a los desafíos o retos cotidianos que le presenta el trabajo.

Siguiendo a Millán y D’Aubeterre (2012), el flujo en el trabajo consta de los siguientes componentes: (1) sentido de control, es decir, el grado en que el trabajador percibe que domina y controla las tareas que comúnmente desempeña; (2) implicación sobre la actividad laboral, supone el grado de satisfacción intrínseca que le produce al trabajador el desempeño de su rol; (3) sensación de unión entre acción y conciencia, referida al grado en que el trabajador percibe una mayor automatización de su ejecución en el trabajo; (4) sentido alterado del tiempo, el cual se refiere a una distorsión en la percepción del paso del tiempo, ya sea como más acelerado o más lento, debido a la alta intensidad de focalización atencional que le dedica el trabajador a la tarea que ejecuta; y (5) sensación de funcionamiento óptimo, la percepción que tiene el trabajador de que sus objetivos personales y los logros obtenidos en el trabajo se encuentran alineados, gracias a que este concibe un plan de acción previo y mantiene una constante retroalimentación sobre su ejecución.

Existen pocos estudios sobre el flujo entre el personal de atención médica (e.g., Leibovich \& Maglio, 2012), los cuales coinciden en afirmar que los entornos laborales de 
atención de salud parecen tener muchas características positivas y posibilitan la experiencia de fluir en el trabajo. Así que, a pesar de las diversas fuentes de estrés laboral, también el personal sanitario puede llegar a disfrutar de las actividades en las que eligió desempeñarse (Bringsén et al., 2012; Nilsson et al., 2005). La explicación de este hecho podría girar alrededor de factores de personalidad (Csikszentmihalyi, 2007) y motivacionales (Bringsén et al., 2011), según los cuales las experiencias de flujo se producen con mayor intensidad en individuos autotélicos (orientados intrínsecamente) que perciben el trabajo como un desafío. Además, en el caso particular de las enfermeras, es probable que los estados de flujo se potencien debido a los objetivos altruistas de ayuda y cuidado con los que el trabajo se asocia (Burke et al., 2016).

Las también denominadas experiencias óptimas se han relacionado con la felicidad, la afectividad positiva, la satisfacción con la vida y el bienestar psicológico (Bryce \& Haworth, 2002). Lo que es más, se las ha vinculado con la salud en general (Fredrickson, 1998). Los estudios también indican que los trabajadores con niveles más altos de flujo muestran mayor satisfacción en el trabajo y mayor eficacia profesional (Fullagar \& Kelloway, 2009). Incluso, se ha probado que, tal como era postulado teóricamente (Seligman, 2011), la disposición a fluir predice el bienestar psicológico y funciona como mediadora de la relación entre este y las condiciones desfavorables de trabajo que pueden atentar contra la salud psicológica (Anaya \& Salanova, 2012; Inciarte \& Morchain, 2011; Millán et al., 2014). Asimismo, investigaciones con enfermeras determinaron que el flujo en el trabajo se relaciona con el afecto positivo y con varios indicadores de rendimiento laboral (Burke et al., 2016), además de cumplir un papel atenuador en los síntomas psicosomáticos (Zito et al., 2015).

En lo adelante se hará referencia a las relaciones del perfeccionismo con los otros constructos. Como se adelantaba, cualquier revisión de la red nomológica del perfeccionismo informa de sus relaciones con variables relativas al malestar psicológico. Sin embargo, algunos autores se han referido a un perfeccionismo normal, positivo, saludable/no patológico o adaptativo (Stoeber \& Otto, 2006). En tal sentido, se ha asegurado que, dentro de ciertos límites, los pensamientos perfeccionistas pueden llegar ser predictores de éxito y bienestar porque se relacionan con el esfuerzo para el logro y el deseo de superación (Blatt, 1995). De modo que el perfeccionismo, así entendido, se asocia con aspectos saludables como la organización, la disciplina, el afrontamiento adaptativo, el afecto positivo y los logros académicos (Fedewa et al., 2005; Frost et al., 1993; Klibert et al., 2005). Esta concepción ha recibido considerable apoyo empírico, a 
partir del cual varios instrumentos que evalúan perfeccionismo han prestado atención también a su faceta más "sana". La preferencia por el orden, dimensión incluida en el modelo de Prieto y Vento (2012), es una de las que representa el aspecto positivo del perfeccionismo.

Cuando la variable considerada es propiamente el bienestar psicológico, los estudios sobre la relación entre esta y el perfeccionismo muestran resultados encontrados. En algunos se consiguió una relación positiva entre ambas variables (e.g., Alim et al., 2016), en otros la relación hallada fue negativa (e.g., Słodkowska \& Bokszczanin, 2012), pero en la mayoría de las investigaciones la relación establecida se diferenció según el tipo de perfeccionismo evaluado: el perfeccionismo saludable o adaptativo tuvo una relación positiva con el bienestar psicológico, mientras que el perfeccionismo no saludable o desadaptativo exhibió una relación negativa con el bienestar (Abbasi et al., 2018; Anand \& Nagle, 2016; Geranmayepour \& Besharat, 2010; Kakavand \& Andish, 2016; Kanten \& Yesiltas, 2015). En esta línea, trabajos de investigación con enfermeras constatan que altos niveles de perfeccionismo negativo se relacionan con estrés laboral, agotamiento profesional y síntomas depresivos (Chang, 2012, 2017; O’Brien \& Page, 1994).

Por otra parte, la relación perfeccionismo-flujo se ha examinado en distintos ámbitos, como el deportivo (Fazlagić \& Belić, 2017), el académico (Ljubin-Golub et al., 2018) y el artístico (Marin \& Bhattacharya, 2013). Siendo la disposición a fluir un constructo salutogénico, se esperaría que, como sucedió en el estudio de Ljubin-Golub et al. (2018), se relacionara positivamente con el perfeccionismo saludable y negativamente con el desadaptativo. Estudios han indicado que el perfeccionismo adaptativo está relacionado con el esfuerzo que se le asigna a una tarea (Stoeber, 2012), por eso es factible suponer que también se relaciona con la inmersión mental o absorción psicológica característica de las experiencias de flujo. Además, como lo sugieren Marin y Bhattacharya (2013), es posible que el flujo sea el responsable de la persistencia que muchos profesionales (e.g., los músicos) muestran a través de la práctica sostenida durante largo tiempo con el objetivo de perfeccionar el desempeño.

Ahora bien, al parecer también es posible que variables como adicción al trabajo y/o pasión obsesiva, sobre las que paradójicamente ya ha podido demostrarse algún vínculo con disposición a fluir (Aponte, 2013; Barahona \& Lisi, 2015), sirvan de puente a la relación entre esta última y perfeccionismo. Varios investigadores se han referido al papel del flujo en los estados de adicción psicológica al trabajo (Del Líbano et al., 2006). 
Según lo plantearon, el inicio de la adicción sería producto de las experiencias positivas del fluir que se generan durante la jornada laboral, sensaciones que llevarían a implicarse más y asumir una mayor dedicación en el trabajo, hasta que finalmente se instala la adicción, de donde se deduce que habría que reconsiderar la capacidad de fluir como un constructo vinculado de forma irrestricta al bienestar.

Explican Aponte y Millán (2013) que cuando una persona posee alto grado de perfeccionismo puede que le resulte más difícil establecer una distancia emocional segura de su trabajo, facilitándose una vinculación más bien adictiva/obsesiva con el mismo y conllevándole esta situación cierta pérdida de bienestar psicológico. Dada la caracterización del perfeccionismo ofrecida por Aponte (2013), parecería que la implicación en el trabajo como adicción se viabiliza ante una estructura de personalidad perfeccionista desadaptativa. Sin embargo, la evidencia apunta a una relación inversa entre el perfeccionismo negativo y el flujo, ya que se supone que el esquema autocrítico que presentan los perfeccionistas desadaptativos no permite que disfruten de lo que hacen y les mantiene sumidos en un estado de ansiedad (De Rosa, 2012) que ha sido tenido como opuesto al flujo (Jackson et al., 1998).

Hechas las consideraciones anteriores, se realizó el presente estudio con el objetivo de evaluar la relación entre el perfeccionismo, el bienestar psicológico y la disposición a fluir en enfermeras de la Gran Caracas; se hizo hincapié en describir las relaciones del perfeccionismo con los otros dos constructos. Obviamente, dicho objetivo supuso haber indagado la incidencia de las mencionadas variables en la muestra de enfermeras estudiada.

El estudio emprendido se justificó en atención a que se podría conocer en mayor medida el comportamiento de las variables y la relación entre ellas. Muy especialmente, la información que se obtuviese podría servir para revisar el papel del perfeccionismo como atributo vinculado o no a la salud psicológica. Hallazgos que además deben valorarse en el marco del que constituye el primer estudio empírico sobre perfeccionismo en una muestra ocupacional venezolana.

\section{Método}

\section{Tipo de investigación}

Se trató de un estudio cuantitativo con alcance descriptivo-correlacional y diseño transversal (Hernández et al., 2014). 


\section{Participantes}

El estudio se realizó con 150 enfermeras residenciadas en la denominada Gran Caracas, con edades comprendidas entre los 22 y los 66 años $(M=41.95, D E=11.243)$, seleccionadas de manera intencional (Kerlinger \& Lee, 2002). El $78 \%(n=117)$ de ellas también había nacido en la Gran Caracas, mientras que el $22 \%(n=33)$ tenía por lugar de procedencia algún otro estado del país. La mayoría se encontraba soltera ( $n=69,46$ $\%)$, con uno $(n=35,23.3 \%)$ o dos $(n=42,28 \%)$ hijos, había alcanzado un nivel educativo universitario $(n=135,90 \%)$, no tenía una profesión adicional a la enfermería $(n=137,91.3 \%)$, tenía un solo trabajo $(n=108,72 \%)$, laboraba en instituciones públicas ( $n=98,65.3 \%)$, en más de un turno $(n=67,44.7 \%) \mathrm{y}$, sobre todo, lo hacía en el área de atención primaria $(n=82,54.6 \%)$. En la tabla 1 , que a continuación se presenta, se pueden apreciar con mayor detalle las características de la muestra.

Tabla 1

Caracterización de la muestra

\begin{tabular}{|c|c|c|c|c|c|}
\hline & $n$ & $\%$ & & $n$ & $\%$ \\
\hline Edad & & & Nivel educativo & & \\
\hline 20-30 años & 29 & 19.3 & Bachillerato & 15 & 10.0 \\
\hline 31-39 años & 41 & 27.3 & T.S.U. & 46 & 30.7 \\
\hline 40-48 años & 29 & 19.3 & Licenciatura & 77 & 51.3 \\
\hline 49-57 años & 33 & 22.0 & Maestría & 12 & 8.0 \\
\hline 58-66 años & 18 & 12.0 & Profesión adicional & & \\
\hline Lugar de nacimiento & & & $\mathrm{Si}$ & 13 & 8.7 \\
\hline Gran Caracas & 117 & 78.0 & No & 137 & 91.3 \\
\hline Interior del país & 33 & 22.0 & Cantidad de trabajos & & \\
\hline Estado civil & & & Uno & 108 & 72.0 \\
\hline Soltera & 69 & 46.0 & Dos & 42 & 28.0 \\
\hline Casada/Unida & 58 & 38.6 & Tipo de institución & & \\
\hline Viuda & 7 & 4.7 & Pública & 98 & 65.3 \\
\hline Divorciada & 16 & 10.7 & Privada & 43 & 28.7 \\
\hline Número de hijos & & & Ambas & 9 & 6.0 \\
\hline Ninguno & 33 & 22.0 & Turno de trabajo & & \\
\hline Uno & 35 & 23.3 & Matutino & 52 & 34.6 \\
\hline Dos & 42 & 28.0 & Vespertino & 24 & 16.0 \\
\hline Tres & 27 & 18.0 & Nocturno & 7 & 4.7 \\
\hline Cuatro & 7 & 4.7 & Más de un turno & 67 & 44.7 \\
\hline \multirow[t]{4}{*}{ Cinco } & 6 & 4.0 & Área de servicio & & \\
\hline & & & Atención primaria & 82 & 54.6 \\
\hline & & & Áreas críticas & 40 & 26.7 \\
\hline & & & Ambas & 28 & 18.7 \\
\hline
\end{tabular}




\section{Instrumentos}

En el desarrollo de este estudio, fueron empleados los siguientes instrumentos de autorreporte:

Escala Multidimensional de Perfeccionismo Abreviada.

Es el producto obtenido por Prieto y Vento (2012) de la validación en Venezuela de la versión española — adaptada por Carrasco et al. (2010) — de la Escala Multidimensional de Perfeccionismo (MPS, por sus siglas en inglés) de Frost et al. (1990). El análisis cualitativo y cuantitativo de los ítems condujo a modificar la redacción de algunos y permitió desechar aquellos que no cumplieron criterios técnicos de calidad. Tras comprobar mediante pruebas estadísticas la pertinencia del procedimiento, se realizaron cuatro análisis factoriales exploratorios, el último de los cuales reportó la solución más satisfactoria, explicando $54.34 \%$ de la varianza total y reteniendo 14 ítems del modelo original, agrupados en tres factores: (1) Preocupación por los Errores (6 ítems, e.g., "Si fallo en el trabajo soy un fracaso como persona"), (2) Organización (4 ítems, e.g., "La organización es muy importante para mí") y (3) Expectativas Paternas (4 ítems, e.g., "Mis padres querían que yo fuese la mejor en todo"). Un análisis factorial confirmatorio ratificó el modelo expuesto como el de mejor ajuste. El instrumento consta de cinco opciones de respuesta tipo Likert (de $1=$ en total desacuerdo a $5=$ completamente de acuerdo). Con respecto a la validez convergente, se evidenciaron correlaciones positivas del perfeccionismo con medidas de afecto negativo y ansiedadrasgo. En cuanto a la validez divergente, se obtuvieron correlaciones inversas entre el perfeccionismo y medidas de afecto positivo y de bienestar psicológico. La confiabilidad de consistencia interna de la escala total, determinada mediante la estimación del coeficiente alfa de Cronbach, fue de .77. Todos los estudios psicométricos se llevaron a cabo con una muestra multiocupacional de trabajadores venezolanos del sector formal de la economía.

Escala de Bienestar Psicológico (EBP).

Se trata de la segunda edición de la EBP elaborada por Sánchez-Cánovas (2007) y validada en Venezuela por Millán y D’Aubeterre (2011). El instrumento evalúa el grado de felicidad y satisfacción con la vida que la persona percibe en su vida cotidiana, a partir 
de 65 ítems distribuidos en cuatro subescalas: (1) Bienestar Subjetivo (30 ítems, e.g., “Acostumbro a ver el lado favorable de las cosas"), (2) Bienestar Material (10 ítems, e.g., “Tengo lo necesario para vivir"), (3) Bienestar Laboral (10 ítems, e.g., "Mi trabajo es creativo, variado, estimulante") y (4) Bienestar en las Relaciones de Pareja (15 ítems, e.g., "Me siento feliz como esposo/a"). En el estudio reportado en el presente artículo se utilizaron las tres primeras subescalas, las cuales emplean un sistema de respuesta tipo Likert de cinco puntos (desde $1=$ nunca, hasta $5=$ siempre). A través de un Análisis de Componentes Principales (ACP) con rotación Varimax se demostró una alta correspondencia entre la estructura factorial original y la obtenida en el contexto laboral venezolano. Justamente, respecto a los indicadores de validez en muestras de trabajadores venezolanos, se han informado correlaciones positivas con disposición a fluir, eficacia profesional, pasión armónica, afrontamiento racional de estresores, cuatro de los cinco grandes rasgos de la personalidad (salvo neuroticismo), afecto positivo, inteligencia emocional, justicia organizacional, diversas fortalezas del carácter (e.g., gratitud) e indicadores de estilos de vida saludables; en contraste, se han reportado correlaciones negativas con medidas de trastornos psicológicos, afecto negativo, cinismo, desgaste emocional, percepción de estrés, neuroticismo, algunas fuentes de estrés laboral, afrontamiento emocional y adicción al trabajo (Aponte, 2013; Arteaga, 2015; Barahona \& Lisi, 2015; Bonilla, 2015; Carruyo \& Leal, 2016; De Ornelas, 2012; Forteza \& González, 2014; García \& Silva, 2016; García \& Ollarves, 2014; Inciarte \& Morchain, 2011; Millán \& D’Aubeterre, 2011; Morchain, 2011; Siblesz \& Von der Heyde, 2013; Suárez, 2014). En el caso de la validación venezolana, el análisis de confiabilidad de la EBP ofreció como resultados un coeficiente alfa de .92 para la escala total, .93 para Bienestar Subjetivo, .91 para Bienestar Material y .81 para Bienestar Laboral.

\section{Escala de Disposición a Fluir en el Trabajo (EDFT-3).}

Este instrumento mide el grado en que un trabajador se encuentra en un estado psicológico óptimo, en términos de la motivación y el disfrute personal de la tarea, en el desempeño de su rol laboral. La escala original fue elaborada a partir de una serie de trabajos dirigidos a valorar el estado de fluidez en el ámbito deportivo. La tercera versión de la EDFT es producto de diversos estudios llevados a cabo por sus autores (Millán \& D’Aubeterre, 2012; Millán et al., 2012), en el marco de la teoría del flujo propuesta por Csikszentmihalyi (2009). Está compuesta por 20 ítems con altos niveles de capacidad 
discriminativa $\left(r_{\mathrm{it}}>.30\right)$ y baja complejidad factorial, es capaz de explicar un porcentaje de varianza superior al $60 \%$, a través de una estructura de cinco factores: (1) Sentido de Control (3 ítems, e.g., "Siento un control total de mi cuerpo y mente mientras trabajo"), (2) Implicación sobre la Actividad Laboral (6 ítems, e.g., "Estar en mi trabajo me hace sentir muy bien"), (3) Unión entre Acción y Conciencia (2 ítems, e.g., "Hago mi trabajo de forma espontánea y automáticamente"), (4) Sentido Alterado del Tiempo (3 ítems, e.g., "Pierdo la noción del tiempo durante el trabajo") y (5) Sensación de Funcionamiento Óptimo (6 ítems, e.g., "En mi trabajo, estoy consciente de lo bien que lo estoy haciendo"). Posteriormente, empleando una metodología confirmatoria se verificó que el modelo pentafactorial exhibe un mejor ajuste frente a modelos rivales (Millán \& D’Aubeterre, 2013). El instrumento se responde a lo largo de una escala Likert de cinco puntos (desde $1=$ mиy en desacuerdo, hasta $5=$ mиy de acuerdo). Estudios con trabajadores venezolanos confirman la presencia de correlaciones positivas entre la EDFT-3 y medidas de bienestar psicológico, inteligencia emocional, responsabilidad como rasgo de personalidad, pasión armoniosa y conductas preventivas que forman parte de un estilo de vida saludable (Aponte, 2013; Arteaga, 2015; Barahona \& Lisi, 2015; Forteza \& González, 2014; García \& Silva, 2016; García \& Ollarves, 2014; Millán \& D’Aubeterre, 2012; Millán et al., 2014). En cambio, en cuanto a correlaciones negativas, destaca la evidenciada por Barahona y Lisi (2015) entre el constructo tratado y la psicopatía. Con respecto a la confiabilidad, Millán et al. (2012) y Millán y D’Aubeterre (2013) señalan que la escala cuenta con indicadores adecuados de consistencia interna, tanto a nivel de la escala total ( $\alpha=.91$ en ambos estudios), como en cada una de sus dimensiones componentes (que oscilaban entre un $\alpha$ de .69 a .87 , y entre .67 a .84 , respectivamente).

\section{Procedimientos}

Para poder llevar a cabo el estudio, se tramitó el permiso correspondiente con la directiva de cada uno de los doce centros de salud, entre públicos y privados, visitados por los investigadores. Aceptada la petición, se acordaron fechas para administrar los instrumentos. Se solicitó la participación voluntaria de las enfermeras, ofreciendo una explicación del objetivo del estudio y garantizando la confidencialidad de los resultados. Una vez obtenido el consentimiento informado por parte de aquellas, se procedió con la aplicación conjunta de los instrumentos. 
El análisis de los datos se realizó con el programa estadístico SPSS, versión 17. Para cada variable del estudio se calculó la media, la desviación estándar y el rango de puntuaciones observadas. Mediante el coeficiente alfa de Cronbach se estimó la consistencia interna de los instrumentos de medición y de sus respectivas dimensiones. Posteriormente, se determinó el nivel de asociación entre las variables estudiadas a través del coeficiente de correlación de Pearson.

\section{Resultados}

\section{Análisis descriptivo}

La tabla 2 presenta los resultados descriptivos de cada variable investigada. Se puede apreciar que, en general, el perfeccionismo de las enfermeras asumió valores medios. Con respecto a los componentes de la conducta perfeccionista, presentaron un nivel bajo de preocupación por los errores, un nivel alto de organización y valores de moderados a altos en cuanto a las expectativas paternas.

Seguidamente, se observa que el nivel de bienestar psicológico de las enfermeras resultó elevado. Lo mismo ocurrió con la percepción del bienestar subjetivo y laboral. Por su parte, en la dimensión relativa al bienestar material se encontraron valores moderados altos.

Por último, se reportaron valores altos de disposición a fluir en el trabajo. El examen del comportamiento estadístico de las dimensiones de esta variable permite informar que las enfermeras presentaron un alto sentido de control, una fuerte implicación sobre la actividad laboral y una elevada sensación de funcionamiento óptimo. Resta decir que la unión entre acción y conciencia, así como el sentido alterado del tiempo, evidenciaron valores entre moderados y altos.

La columna final de la tabla 2 permite apreciar que la mayoría de las medidas exhibió una consistencia interna satisfactoria, con coeficientes por encima del .70 sugerido por Oviedo y Campo-Arias (2005). Asimismo, a pesar del bajo número de ítems con que fueron calculados, los coeficientes de las subescalas Organización y Expectativas Paternas se hallan muy próximos al recomendado ( $\alpha=.68$ y .67, respectivamente).

Por el contrario, en las subescalas Unión entre Acción y Conciencia, Sentido de Control y Sentido Alterado del Tiempo se obtuvieron índices alejados de lo conveniente $(\alpha=.62, .56$ y .53 , respectivamente). Estos resultados pueden explicarse considerando que el coeficiente alfa se ve muy influido por la cantidad de ítems que se incluyen en su 
cálculo y las mencionadas dimensiones son las que agrupan menos ítems (entre dos y tres reactivos).

Tabla 2

Descriptivos e índices de consistencia interna de las medidas utilizadas

\begin{tabular}{|c|c|c|c|c|c|c|}
\hline & $M$ & $D E$ & $\begin{array}{l}\text { Rango } \\
\text { teórico }\end{array}$ & $\begin{array}{c}\text { Rango } \\
\text { observado }\end{array}$ & $\begin{array}{l}\text { Núm. } \\
\text { ítems }\end{array}$ & $\alpha$ \\
\hline 1. Perfeccionismo & 44.49 & 6.53 & $14-70$ & $27-60$ & 14 & .71 \\
\hline 2. Preocupación por los & 12.26 & 4.22 & $6-30$ & $6-27$ & 6 & .73 \\
\hline 3. Organización & 17.77 & 1.98 & $4-20$ & $12-20$ & & .68 \\
\hline 4. Expectativas paternas & 14.47 & 3.35 & $4-20$ & $4-20$ & 4 & .67 \\
\hline 5. Bienestar psi & 219.87 & 21.09 & $50-250$ & $127-250$ & 50 & .93 \\
\hline 6. Bienestar subjetivo & 133.88 & 12.28 & $30-150$ & $95-150$ & 30 & .89 \\
\hline 7. Biene & 41.41 & 7.49 & $10-50$ & & 10 & .93 \\
\hline 8. $\mathrm{Bi}$ & 44.58 & 4.78 & $10-50$ & 19 & 10 & .73 \\
\hline 9. Dis & 81.31 & 8.39 & $20-100$ & $54-100$ & 20 & .85 \\
\hline 10. Sentido de con & 12.97 & 1.80 & $3-15$ & $6-15$ & 3 & .56 \\
\hline $\begin{array}{l}\text { 11. Implicación sobre la actividad } \\
\text { laboral }\end{array}$ & 77 & 50 & 30 & 1 & 6 & .78 \\
\hline 12. Unión entre acción y concienci & 1.90 & 1.91 & & $2-10$ & 2 & .62 \\
\hline 13. Sentic & 10.98 & 2.50 & $3-15$ & $5-15$ & 3 & .53 \\
\hline 14. Sensación de funcionamiento óptimo & 26.51 & 2.92 & $6-30$ & $16-30$ & 6 & .84 \\
\hline
\end{tabular}

\section{Análisis correlacional}

La tabla 3 presenta los resultados de las asociaciones entre las variables medidas. Se observa que el perfeccionismo presentó correlaciones positivas con el bienestar psicológico y dos de sus facetas: el bienestar subjetivo y el material $(p<.05 ; p<.01)$. Por su parte, la organización es un factor constituyente del perfeccionismo que se vinculó positivamente con el bienestar psicológico y sus respectivas dimensiones $(p<.01)$. El mismo patrón se cumplió con las expectativas paternas, solo que en este caso las correlaciones fueron un poco más bajas, aunque igualmente significativas $(p<.01)$. Cabe acotar que la preocupación por las equivocaciones no correlacionó con el bienestar, ni con ninguna de sus dimensiones.

Puede también observarse que el perfeccionismo mostró correlaciones positivas con la disposición a fluir en el trabajo y sus dimensiones $(p<.01)$. Las expectativas paternas exhibieron un patrón similar. En el caso de la organización, esta correlacionó de manera positiva con la disposición a fluir y tres de sus componentes, a saber: el sentido de control, la implicación sobre la actividad laboral y la sensación de funcionamiento óptimo $(p<.01)$. Además, vale destacar que la preocupación por los errores no se asoció con la disposición a fluir en ninguna de sus dimensiones. 
Continuando con el examen visual de la matriz de correlaciones, se aprecia que la disposición a fluir en el trabajo tuvo asociaciones positivas con el bienestar psicológico y todas sus facetas $(p<.01)$. De igual manera, la implicación sobre la actividad laboral y la sensación de funcionamiento óptimo se asociaron positivamente con el bienestar y sus tres dimensiones $(p<.01)$. En cambio, el sentido de control presentó correlaciones positivas con el bienestar psicológico y dos de sus facetas, el bienestar subjetivo y el laboral $(p<.05 ; p<.01)$. Por su parte, la unión entre acción y conciencia, además de evidenciar correlaciones con el bienestar psicológico como un todo, únicamente mostró una relación positiva con el componente subjetivo del bienestar $(p<.05)$. Por último, es pertinente señalar que el sentido alterado del tiempo no se vinculó ni con el bienestar psicológico, ni con alguna de sus dimensiones.

Tabla 3

Correlaciones entre perfeccionismo, bienestar psicológico y disposición a fluir en el trabajo

\begin{tabular}{|c|c|c|c|c|c|c|c|c|c|c|c|c|c|}
\hline & 12 & 232 & 4 & 5 & 6 & 7 & 8 & 9 & 10 & 11 & 12 & 13 & 14 \\
\hline 1. Perfeccionismo & 1 & & & $.22 * *$ & $.22 * *$ & $.19^{*}$ & & $.38^{* *}$ & $.26 * *$ & $.21 * *$ & $24 * *$ & $.25 * *$ & $.39 * *$ \\
\hline $\begin{array}{l}\text { 2. Preocupación por los } \\
\text { errores }\end{array}$ & 1 & 1 & & & & & & & & & & & \\
\hline 3. Organización & & 1 & & $.43 * *$ & $.39 * *$ & $.39 * *$ & $.30 * *$ & $.33 * *$ & $.34 * *$ & $.26 * *$ & & & $.36^{* *}$ \\
\hline $\begin{array}{l}\text { 4. Expectativas } \\
\text { paternas }\end{array}$ & & & 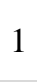 & $.33 * *$ & $.30 * *$ & $.31 * *$ & $.21 * *$ & $.44 * *$ & $.33^{* *}$ & $.31 * *$ & $.24 * *$ & $.27 * *$ & $.42 * *$ \\
\hline $\begin{array}{l}\text { 5. Bienestar } \\
\text { psicológico }\end{array}$ & & & & 1 & & & & $.40 * *$ & $.26 * *$ & $.39 * *$ & $.19 *$ & & $.46^{* *}$ \\
\hline 6. Bienestar subjetivo & & & & & 1 & & & $.44 * *$ & $.29 * *$ & $.40 * *$ & $.20 *$ & & $.49 * *$ \\
\hline 7. Bienestar material & & & & & & 1 & & $.25 * *$ & & $.26^{* *}$ & & & $.30 * *$ \\
\hline 8. Bienestar laboral & & & & & & & 1 & $.26^{* *}$ & $.18^{*}$ & $.32 * *$ & & & $.30 * *$ \\
\hline $\begin{array}{l}\text { 9. Disposición a fluir } \\
\text { en el trabajo }\end{array}$ & & & & & & & & 1 & & & & & \\
\hline 10. Sentido de control & & & & & & & & & 1 & & & & \\
\hline $\begin{array}{l}\text { 11. Implicación sobre } \\
\text { la actividad laboral }\end{array}$ & & & & & & & & & & 1 & & & \\
\hline $\begin{array}{l}\text { 12. Unión entre acción } \\
\text { y conciencia }\end{array}$ & & & & & & & & & & & 1 & & \\
\hline $\begin{array}{l}\text { 13. Sentido alterado } \\
\text { del tiempo }\end{array}$ & & & & & & & & & & & & 1 & \\
\hline $\begin{array}{l}\text { 14. Sensación de } \\
\text { funcionamiento óptimo }\end{array}$ & & & & & & & & & & & & & 1 \\
\hline $\begin{array}{l}\text { Nota: } N=150 . \\
* p<.05 . * * p<.01 .\end{array}$ & & & & & & & & & & & & & \\
\hline
\end{tabular}

\section{Discusión}

El objetivo de este estudio ha sido analizar la relación entre las variables perfeccionismo, bienestar psicológico y disposición a fluir en un grupo de enfermeras que trabaja en centros de salud públicos y privados de la Gran Caracas. Naturalmente, tal objetivo implicó haber dado cuenta del nivel que alcanzan esas variables en la muestra examinada. 
Los niveles moderados de perfeccionismo aunados a la elevada valoración del orden evidencian la relevancia del constructo en las enfermeras, tal como lo señalaba la literatura citada en la introducción. Contrario a lo esperado, el grupo exhibió poca preocupación por las equivocaciones. Esto llama la atención porque se supone que las enfermeras, además de ser organizadas y pulcras, deben ejercer su trabajo con cautela y precisión, evitando cometer errores que puedan poner en riesgo la salud de los pacientes que atienden (Chang, 2012; Melrose, 2011). Sin embargo, dicho resultado podría explicarse tomando en cuenta que la mayor parte de la muestra trabaja en áreas de atención primaria (i.e., consulta externa, laboratorio) y no en áreas críticas (i.e., hospitalización, cirugía, terapia intensiva, emergencia), siendo probable que la meticulosidad con que las enfermeras del estudio desempeñan sus labores sea un aspecto menos importante e incurrir en un error no signifique comprometer la vida del paciente.

Por otro lado, las enfermeras perciben que sus padres les han puesto metas elevadas durante sus vidas, percepción que quizá sigue guiando el actuar de estas mujeres. Este es otro hallazgo que llama la atención pues, en principio, se pensaría que las demandas parentales, ya sean reales o percibidas, juegan un papel más activo en la conducta de los niños y no tanto en el comportamiento de las personas de edad madura. Es por ello que autores como Rhéaume et al. (1995) consideraron que las críticas paternas son un aspecto que contribuye poco a la comprensión de la conducta perfeccionista. Años más tarde, en la búsqueda de una medida que incluyera los aspectos esenciales del perfeccionismo, Hill et al. (2004) observaron que la subescala Presión Parental Percibida no se agrupó en ninguno de los factores de segundo orden que delimitaron. Incluso en adolescentes chinos y mexicanos de ambos sexos, el factor Críticas Paternas (hermanado con el factor Expectativas Paternas) de la MPS no se conformó (Cheng et al., 1999; Franco et al., 2010), hecho que da a entender que los jóvenes de estas nacionalidades perciben pocas críticas por parte de sus padres.

Ahora bien, en el caso venezolano, es importante tener en cuenta que la mujer cumple con muchos roles a la vez, no solo vive tratando de satisfacer sus necesidades y las de sus hijos, sino que también está pendiente de las necesidades de sus padres, a las cuales trata de dar cobertura (Pinto \& Rodríguez, 2015). Justamente, parte de la explicación podría radicar en diferencias en cuanto a la forma en que la opinión y los comentarios de los padres son percibidos por hombres y mujeres. Por ejemplo, en México, algunas investigaciones han señalado que en los hombres la opinión de los demás, particularmente la de sus padres, no influye de manera importante, mientras que las 
mujeres valoran más sus logros en función de la opinión de los demás (Mercado \& Luna, 2003). De hecho, en el análisis psicométrico de la MPS en población mexicana, las subescalas Expectativas Paternas y Críticas Paternas se conformaron únicamente en el caso de las mujeres (Franco, 2007), lo que de nuevo sugiere diferencias de género en cuanto a los componentes del perfeccionismo.

Habida cuenta de las múltiples situaciones estresantes a las que se supone están expuestas las enfermeras, los elevados niveles de bienestar psicológico reportados por la muestra merecen algunos comentarios. En primer lugar, se debe tener presente que, dado el carácter de valoración subjetiva de los elementos evaluados en la EBP, este instrumento es sensible a la percepción que la persona tiene de sus circunstancias vitales y no necesariamente a su calidad de vida o salud mental en términos objetivos. Por ejemplo, mediante la dimensión Bienestar Material se tiene acceso a la percepción subjetiva de la persona sobre sus condiciones materiales, con independencia de cómo estas sean en realidad. También se ha verificado que, aunque con medias hacia el extremo superior de la escala de respuesta, la mitad de los ítems del factor Bienestar Material se encuentran entre los que obtuvieron las medias más bajas de la distribución, por cierto se trata de los ítems más representativos de la dimensión, aluden a la seguridad económica a largo plazo (ítems 40, 36 y 39) y al hecho de vivir con comodidades (ítems 37 y 38); en contraste, los ítems más contestados con la opción siempre son los que hacen referencia a sentirse "afortunada" (ítem 32), llevar "una vida tranquila" (ítem 33), tener "lo necesario" (ítem 34) y "vivir con cierto desahogo" (ítem 31), aspectos que semántica y culturalmente no se circunscriben solo al ámbito de lo material (e.g., en Venezuela, alguien que se considera "afortunado" puede que simplemente piense que le han pasado cosas buenas o que tiene suerte).

En segundo lugar, es posible que el impacto del estrés que afecta a las enfermeras se vea mitigado e incluso contrarrestado por la satisfacción intrínseca que produce el trabajo de atención en salud, sumada a la acción de ciertas variables protectoras (e.g., optimismo, humor, resiliencia), algunas de las cuales se encuentran siendo investigadas activamente desde la psicología positiva como fortalezas que caracterizan a los venezolanos (e.g., Garassini \& Camilli, 2012). En este trabajo, y a modo exploratorio, se realizó un ACP de la dimensión Bienestar Subjetivo (utilizando el criterio de eigenvalue $>1$ y rotación varimax), a partir del cual se pudo observar que sus ítems se agruparon en familias temáticas, de manera que varios de los que tuvieron las medias más altas (y, por tanto, aquellos en los que descansa el bienestar de la muestra) también se reunieron en un 
mismo factor: es el caso de ítems como el 24 ("Me gusta lo que hago") y el 22 ("Tengo muchas ganas de vivir"), los cuales podrían apuntar al sentido (meaning) postulado por Seligman (2011) como un componente del bienestar de las personas; lo mismo sucedió con los ítems 30 ("Tengo confianza en mí misma") y 19 ("Creo que puedo superar mis errores y debilidades"), en los que parece operar la autoestima y el logro como factores protectores.

Por lo que se refiere a la disposición a fluir, las enfermeras informaron de un elevado sentido de control sobre las tareas que cotidianamente les demanda el trabajo, una fuerte implicación con sus ocupaciones, misma que denota el carácter autotélico (intrínseco) de la motivación que les guía, y una sensación intensa de funcionamiento óptimo durante la ejecución de sus actividades. En un estudio con trabajadores del sector salud de Caracas, Cartolano et al. (2011) consiguieron niveles de flujo considerablemente elevados en la muestra. Según ellos mismos lo explican, es probable que ocupaciones como las del área de la salud produzcan satisfacción en sus trabajadores por el hecho de que el resultado de un buen desempeño redunda de manera más o menos directa, inmediata y palpable en el usuario de la atención. Al parecer, a diferencia del trabajo administrativo, aquel que implica un contacto directo con el otro, como el de los docentes y profesionales de la salud, puede entrañar — de por sí — un significado más trascendental para quienes lo ejercen. Tan es así que tal satisfacción laboral competiría incluso con los estresores que frecuentemente deben afrontar estos empleados. De hecho, de acuerdo con Csikszentmihalyi (2007), auténticas experiencias de flujo pueden tener lugar hasta en los ambientes más hostiles y esto sucede porque hay trabajadores para quienes un contexto adverso puede representar un reto y el solo hecho de asumirlo pasa a constituir una gran recompensa.

La sensación de unión acción-conciencia al desarrollar el trabajo y el sentido alterado del tiempo mostraron niveles un poco menos elevados. En cuanto a la primera dimensión, se podría suponer que las enfermeras ejecutan tareas que, dada su complejidad, no llegan a automatizarse del todo; en ese tipo de tareas es posible que, más que la velocidad en la ejecución, lo realmente importante sea la calidad de la misma, evidenciada en una atención personalizada que marque la diferencia. Algo parecido sucede con respecto a la percepción del tiempo, pues por más disfrute que implique el trabajo es difícil pensar en una distorsión temporal total en el caso de las enfermeras, quienes ejecutan tareas (e.g., la administración de medicamentos cada cierto tiempo, la estimación de la duración de un procedimiento médico, el control de citas de los 
pacientes) en las que es necesario tener una percepción bastante cercana a la realidad del tiempo transcurrido (Kozier et al., 2008).

En consonancia con lo reportado en la literatura, el bienestar psicológico se asoció positivamente con la disposición a fluir en el trabajo. El hecho de que el bienestar material no se asociara con el sentido de control indica que la experticia que una enfermera despliega en su trabajo no guarda relación con la mejoría de la situación económica que vive. Esta es una aseveración que pone de relieve la posible existencia de un sistema laboral anómalo, en el cual la calidad del desempeño de los empleados no va de la mano con —en el sentido de que "no tiene el poder de influir sobre" — las ganancias económicas que perciben. Así mismo, trabajar "de forma automática" no hace que mejoren las finanzas personales. Incluso, puede que las enfermeras obtengan peores resultados si trabajan de manera precipitada o por salir del paso, de allí que el trabajo automatizado no haya correlacionado tampoco con el bienestar laboral. Por su parte, el sentido alterado del tiempo no se asoció con el bienestar psicológico. Un resultado similar obtuvieron García y Silva (2016) con una muestra de trabajadores de empresas públicas y privadas de Caracas. Quizás sea necesario clarificar muy bien el papel de esta dimensión del flujo, ya que muchas veces la distorsión en la percepción del tiempo ha sido tratada como síntoma de trastorno mental (e.g., estrés postraumático, demencia) o como consecuencia de la acción de sustancias psicoactivas (Gutiérrez-García et al., 2017).

El perfeccionismo general se relacionó positivamente con el bienestar psicológico. Este hallazgo, en principio, viene a refutar la visión negativa y dominante del perfeccionismo, además de contradecir los resultados del estudio de convergencia/divergencia sobre la escala abreviada de Prieto y Vento (2012). Igualmente, un alto nivel de organización se asoció con mayor bienestar, confirmándose así que la necesidad de orden es uno de los componentes del perfeccionismo que, en sí mismo, no interfiere con el funcionamiento adaptativo y normal (Stoeber \& Otto, 2006), sino que puede promover la expansión de conocimientos, los buenos hábitos de trabajo, el esfuerzo y el buen desempeño (Blatt, 1995). En la investigación de Butt (2010) se obtuvo un resultado similar al expuesto: la organización tuvo una relación positiva con el bienestar psicológico y todas sus dimensiones, además el grupo de participantes con mayores niveles de organización mostró puntajes significativamente más altos en bienestar y en tres de sus dimensiones (dominio del entorno, autoaceptación y crecimiento personal).

De forma inesperada, también se evidenció una correlación positiva entre las expectativas de los padres y el bienestar psicológico. Tales expectativas tradicionalmente 
se vinculan a la disfunción psicológica porque se trata de percepciones, es decir, no necesariamente son ciertas y, en la medida en que son irreales y exageradas, se hacen imposibles de satisfacer. De hecho, a menudo las expectativas paternas se han identificado con el perfeccionismo socialmente prescrito postulado por Hewitt y Flett (1991), y ambas manifestaciones son parte de las llamadas preocupaciones perfeccionistas, las cuales han sido asociadas con indicadores de desajuste psicológico (Stoeber \& Otto, 2006). A pesar de lo sorpresivo del resultado, también en el estudio de Butt (2010) las expectativas paternales correlacionaron positivamente con el bienestar y con dos de sus dimensiones, el dominio del entorno y el propósito en la vida; además, quienes puntuaron alto en expectativas paternas, obtuvieron altas puntuaciones en bienestar psicológico, dominio del entorno, propósito en la vida y autoaceptación. Para el caso de las enfermeras, enmarcado en el contexto venezolano, se cree que las expectativas por parte de sus padres son interpretadas de manera positiva debido a que consideran que son una muestra de atención y afecto, de modo tal que la conducta dirigida a complacer estas demandas sería generadora de un profundo sentimiento de orgullo y valía personal.

La bibliografía especializada se ha referido a la preocupación ante la posibilidad de cometer errores como la característica esencial del perfeccionismo no saludable, por estar fuertemente relacionada con diversas formas de psicopatología (Cheng et al., 1999; Franco et al., 2010; Frost et al., 1990; Rhéaume et al., 1995; Stoeber \& Otto, 2006). Un ejemplo son los resultados del estudio realizado por Chang (2012) con enfermeras de Taiwán, en el que la preocupación por las equivocaciones mostró relaciones positivas con desgaste profesional y modos de afrontamiento desadaptativos. En la presente investigación, al igual que en la de Butt (2010), la preocupación por los errores no tuvo una relación significativa con el bienestar psicológico, sin que por ello pueda asegurarse que se asocia con el malestar subjetivo.

Las enfermeras con altos niveles de perfeccionismo reportaron mayor disposición a fluir en el trabajo, resultado que vuelve a señalar al perfeccionismo como un rasgo globalmente saludable. Las relaciones de la organización con la fusión acción-conciencia y el sentido alterado del tiempo no alcanzaron significatividad estadística; parece que la sistematicidad es incompatible con la desorientación temporal, al igual que con un trabajo ejecutado de forma automática e inconsciente. Por su parte, la relación entre las expectativas paternas y el flujo ofrece un reto interpretativo, toda vez que dicha asociación fue incluso más fuerte que la dupla expectativas-bienestar. Es posible que, en el contexto local, la entrega que muestran las enfermeras en el trabajo tenga una relación 
directa con la gratificación de las necesidades de los padres, lo que le imprimiría un valor agregado a la actividad laboral; otra posibilidad a considerar es que las operaciones cognitivas que se necesitan para poner en marcha un estado de flujo sean similares a las que se requieren para formarse expectativas percibidas. Finalmente, aunque se esperaba encontrar una relación negativa entre la preocupación por los errores y la fluidez (como la conseguida por Fazlagić \& Belić, 2017), dichas variables no se asociaron en ninguna dirección.

Este trabajo presenta algunas limitaciones, entre ellas el uso exclusivo de medidas de autoinforme en el estudio de las variables psicológicas. Al respecto es preciso destacar que, si bien dicha estrategia es una de las más recomendables para la recogida de datos, puede dar lugar a sesgos sobre la información recibida de las participantes, siendo quizás el caso más ilustrativo el de la medición del bienestar material. También pueden tenerse como limitantes el bajo tamaño muestral y el hecho de no haber seguido un muestreo aleatorio. Futuros estudios deben ampliar el tamaño de la muestra, asegurar la representatividad de la misma, así como tratar de controlar en mayor medida las variables — sociodemográficas y organizacionales - que puedan afectar los datos obtenidos. Otra limitación está referida a la utilización de un diseño correlacional, el cual se reduce al examen de relaciones simples entre variables sin establecer relaciones causales, propósito al que deberían abocarse futuras investigaciones. A pesar de ello, las conclusiones a las que se llegó pueden justificar el diseño de programas de intervención, por ejemplo dirigiéndolos a favorecer rasgos como el orden y la organización en relación con el perfeccionismo, a la vez que se enseña a lidiar con las equivocaciones y la angustia que comporta la posibilidad de cometerlas. 


\section{Referencias}

Abbasi, M., Pirani, Z., \& Salehi, E. (2018). Investigate the relationship between positive and negative perfectionism with emotional well-being and academic engagement in nurse's students. Education Strategies in Medical Sciences, 10(6), 447-454. http://edcbmj.ir/article-1-1189-en.html

Aiken, L., Clarke, S., Sloane, D., Sochalski, J., Busse, R., Clarke, H., Giovannetti, P., Hunt, J., Rafferty, A., \& Shamian, J. (2001). Nurses' reports on hospital care in five countries. Health Affairs, 20(3), 43-53. https://doi.org/10.1377/hlthaff.20.3.43

Alim, A., Rathakrishnan, B., Alim, A., \& Voo, P. (2016). Relationship of perfectionism and basic psychological needs to psychological well-being among university students at Universiti Sabah, Malaysia. Jurnal Psikologi Malaysia, 30(2), 13-20. http://spaj.ukm.my/ppppm/jpm/article/view/223

Anand, Y., \& Nagle, K. (2016). Perfectionism as predictor of psychological wellbeing among college students. Global Journal of Human-Social Science Research, 16(5). https://socialscienceresearch.org/index.php/GJHSS/article/view/1831

Anaya, L., \& Salanova, C. (2012). Disposición a fluir en el trabajo como mediadora entre las condiciones de trabajo y el bienestar psicológico de una muestra multiocupacional del Área Metropolitana [Trabajo final de licenciatura no publicado]. Universidad Metropolitana.

Aponte, E. (2013). Personalidad y disposición a fluir en el trabajo: ¿un camino al bienestar psicológico o a la adicción al trabajo? [Trabajo final de licenciatura no publicado]. Universidad Metropolitana.

Aponte, E., \& Millán, A. (2013). Personalidad y disposición a fluir en el trabajo: ¿un camino al bienestar psicológico o a la adicción al trabajo? Anales de la Universidad Metropolitana, 13(2), 191-210. http://openjournal.unimet.edu.ve/index.php/Anales/article/view/27

Arteaga, L. (2015). Disposición a fluir en el trabajo y bienestar psicológico en trabajadores del Circuito Judicial de Trujillo [Trabajo Especial de Grado no publicado]. Universidad Rafael Urdaneta.

Barahona, S., \& Lisi, S. (2015). Personalidad oscura, disposición a fluir y perfil dual de pasión como factores explicativos del bienestar psicológico y adicción al trabajo [Trabajo final de licenciatura no publicado]. Universidad Metropolitana.

Blatt, S. (1995). The destructiveness of perfectionism. Implications for de treatment of depression. American Psychologist, 50(12), 1003-1020. https://doi.org/10.1037/0003-066X.50.12.1003

Bonilla, C. (2015). Fortalezas del carácter y bienestar psicológico en militares activos de la Fuerza Armada Nacional Bolivariana [Tesis de maestría no publicada]. Universidad Rafael Urdaneta.

Bringsén, Å., Andersson, H., Ejlertsson, G., \& Troein, M. (2012). Exploring workplace related health resources from a salutogenic perspective. Results from a focus group study among healthcare workers in Sweden. Work, 42(3), 403-414. https://doi.org/10.3233/WOR-2012-1356

Bringsén, Å., Ejlertsson, G., \& Andersson, I. (2011). Flow situations during everyday practice in a medical hospital ward. Results from a study based on experience sampling method. BMC Nursing, 10(3), 1-9. https://doi.org/10.1186/1472-6955-10-3 
Bryce, J., \& Haworth, J. (2002). Wellbeing and flow in sample of male and female office workers. Leisure Studies, 21(3-4), 249-263. https://doi.org/10.1080/0261436021000030687

Burke, R. (2003). Length of shift, work outcomes, and psychological well-being of nursing staff. International Journal of Public Administration, 26(4), 16371646. https://doi.org/10.1081/PAD-120024415

Burke, R., Koyuncu, M., \& Fiksenbaum, L. (2016). Flow, work satisfactions and psychological well-being among nurses in Turkish hospitals. Archives of Nursing Practice and Care, 2(1), 10-17. https://doi.org/10.17352/anpc.000007

Butt, F. (2010). The role of perfectionism in psychological health: A study of adolescents in Pakistan. Europe's Journal of Psychology, 6(4), 125-147. https://doi.org/10.5964/ejop.v6i4.227

Carrasco, A., Belloch, A., \& Perpiñá, C. (2010). La evaluación del perfeccionismo: utilidad de la Escala Multidimensional de Perfeccionismo en población española. Análisis y Modificación de Conducta, 36(153), 49-65. http://www.uhu.es/publicaciones/ojs/index.php/amc/article/view/1225

Carruyo, D., \& Leal, I. (2016). Bienestar psicológico y gratitud en el personal administrativo de una institución financiera [Trabajo Especial de Grado no publicado]. Universidad Rafael Urdaneta.

Cartolano, A., Padrón, J., \& Rojas, E. (2011). Inteligencia emocional como mediadora de los estados de flow y bienestar percibido en trabajadores del sector de la salud público y privado en Caracas [Trabajo final de licenciatura no publicado]. Universidad Metropolitana.

Chang, Y. (2012). The relationship between maladaptive perfectionism with burnout: Testing mediating effect of emotion-focused coping. Personality and Individual Differences, 53(5), 635-639. https://doi.org/10.1016/j.paid.2012.05.002

Chang, Y. (2017). Benefits of being a healthy perfectionist: Examining profiles in relation to nurses' well-being. Journal of Psychosocial Nursing and Mental Health Services, 55(4), 22-28. https://doi.org/10.3928/02793695-20170330$\underline{04}$

Chang, Y., Wang, P., Li, H., \& Liu, Y. (2011). Relations among depression, selfefficacy, and optimism in a sample of nurses in Taiwan. Journal of Nursing Management, 19(6), 769-776. https://doi.org/10.1111/j.13652834.2010.01180.x

Cheng, S., Chong, G., \& Wong, C. (1999). Chinese Frost Multidimensional Perfectionism Scale: A validation and prediction of self-esteem and psychological distress. Journal of Clinical Psychology, 55(9), 1051-1061. https://doi.org/10.1002/(SICI)1097-4679(199909)55:93.3.CO;2-T

Craiovan, P. (2014). Correlations between perfectionism, stress, psychopathological symptoms and burnout in the medical field. Procedia - Social and Behavioral Sciences, 127, 529-533. https://doi.org/10.1016/j.sbspro.2014.03.304

Csikszentmihalyi, M. (2007). Fluir (Flow): una psicología de la felicidad (12a ed.). Kairós.

Csikszentmihalyi, M. (2009). El flujo. En E. Fernández-Abascal (Ed.), Emociones positivas (pp. 181-193). Pirámide.

Csikszentmihalyi, M., \& Csikszentmihalyi, I. (1998). Experiencia óptima: estudios psicológicos del flujo en la conciencia. Desclée de Brouwer. 
D’Souza, F., Egan, S., \& Rees, C. (2011). The relationship between perfectionism, stress and burnout in clinical psychologists. Behaviour Change, 28(1), 17-28. https://doi.org/10.1375/bech.28.1.17

De Ornelas, M. (2012). Disposición a fluir en el trabajo, percepción de estrés laboral y bienestar psicológico de una muestra de obreros de productos cárnicos [Trabajo final de licenciatura no publicado]. Universidad Metropolitana.

De Rosa, L. (2012). Factores mantenedores del perfeccionismo desadaptativo o clínico. En A. Grassi (Ed.), Memorias del IV Congreso Internacional de Investigación y Práctica Profesional en Psicología - XIX Jornadas de Investigación - VIII Encuentro de Investigadores en Psicología del MERCOSUR (pp. 39-42). Universidad de Buenos Aires. http://www.aacademica.org/000-072/210.pdf

Del Líbano, M., Rodríguez, A., Llorens, S., Cifre, E., \& Salanova, M. (2006). Adicción al trabajo y flow: una relación conflictiva. Riesgo Laboral, 14, 3437. http://repositori.uji.es/xmlui/handle/10234/18893

Fazlagić, A., \& Belić, M. (2017). The connection of perfectionism and flow with athletes of a different performance level. Fizička kultura, 71(2), 111-117. https://doi.org/10.5937/fizkul1702117F

Fedewa, B., Burns, L., \& Gomez, A. (2005). Positive and negative perfectionism and the shame/guilt distinction: Adaptive and maladaptive characteristics. Personality and Individual Differences, 38(7), 1609-1619. https://doi.org/10.1016/j.paid.2004.09.026

Fiabane, E., Giorgi, I., Sguazzin, C., \& Argentero, P. (2013). Work engagement and occupational stress in nurses and other healthcare workers: The role of organisational and personal factors. Journal of Clinical Nursing, 22(17-18), 2614-2624. https://doi.org/10.1111/jocn.12084

Forteza, M., \& González, A. (2014). Pasión: su papel mediador entre personalidad y disposición a fluir en el trabajo con respecto al bienestar psicológico y la adicción al trabajo [Trabajo final de licenciatura no publicado]. Universidad Metropolitana.

Franco, K. (2007). Perfeccionismo y trastornos del comportamiento alimentario: una aproximación multidimensional [Tesis doctoral no publicada]. Universidad Nacional Autónoma de México.

Franco, K., Mancilla, J., Vázquez, R., Álvarez, G., \& López, X. (2010). Estructura factorial y consistencia interna de la Escala Multidimensional de Perfeccionismo. Revista Mexicana de Psicología, 27(2), 143-149. https://www.redalyc.org/articulo.oa?id=243016324003

Fredrickson, B. (1998). What good are positive emotions? Review of General Psychology, 2(3), 300-319. https://doi.org/10.1037/1089-2680.2.3.300

Fronteira, I., \& Ferrinho, P. (2011). Do nurses have a different physical health profile? A systematic review of experimental and observational studies on nurses' physical health. Journal of Clinical Nursing, 20(17-18), 2404-2424. https://doi.org/10.1111/j.1365-2702.2011.03721.x

Frost, R., Heimberg, R., Holt, C., Mattia, J., \& Neubauer, A. (1993). A comparison of two measures of perfectionism. Personality and Individual Differences, 14(1), 119-126. https://doi.org/10.1016/0191-8869(93)90181-2

Frost, R., Marten, P., Lahart, C., \& Rosenblate, R. (1990). The dimensions of perfectionism. Cognitive Therapy and Research, 14(5), 449-468. https://doi.org/10.1007/BF01172967 
Fullagar, C., \& Kelloway, E. (2009). Flow at work: An experience sampling approach. Journal of Occupational and Organizational Psychology, 82(3), 595-615. https://doi.org/10.1348/096317908X357903

Garassini, M., \& Camilli, C. (2012). La felicidad duradera. Estudios sobre el bienestar en la Psicología Positiva. Alfa.

García, B., \& Silva, K. (2016). La disposición a fluir en el trabajo, estilos de vida saludable y el bienestar psicológico en trabajadores de la ciudad de Caracas [Trabajo final de licenciatura no publicado]. Universidad Metropolitana.

García, D., \& Ollarves, E. (2014). Bienestar psicológico, inteligencia emocional y disposición a fluir en docentes universitarios [Trabajo Especial de Grado no publicado]. Universidad Rafael Urdaneta.

Gardulf, A., Söderström, I., Orton, M., Eriksson, L., Arnetz, B., \& Nordström, G. (2005). Why do nurses at a university hospital want to quit their jobs? Journal of Nursing Management, 13(4), 329-337. https://doi.org/10.1111/j.1365-2934.2005.00537.x

Geranmayepour, S., \& Besharat, M. (2010). Perfectionism and mental health. Procedia Social and Behavioral Sciences, 5, 643-647. https://doi.org/10.1016/j.sbspro.2010.07.158

Gutiérrez-García, A., Reyes-Platas, D., \& Picazo, O. (2017). Percepción del tiempo en la neuropsicopatología: una revisión sistemática. Psiquiatría Biológica, 24(3), 85-96. https://doi.org/10.1016/j.psiq.2017.10.002

Hernández, R., Fernández, C., \& Baptista, P. (2014). Metodología de la investigación ( $6^{\mathrm{a}} \mathrm{ed}$.). McGraw-Hill Interamericana.

Hewitt, P., \& Flett, G. (1991). Perfectionism in the self and social contexts: Conceptualization, assessment, and association with psychopathology. Journal of Personality and Social Psychology, 60(3), 456-470. https://doi.org/10.1037/0022-3514.60.3.456

Hill, R., Huelsman, T., Furr, M., Kibler, J., Vicente, B., \& Kennedy, C. (2004). A new measure of perfectionism: The Perfectionism Inventory. Journal of Personality Assessment, 82(1), 80-91. https://doi.org/10.1207/s15327752jpa8201 13

Inciarte, G., \& Morchain, A. (2011). Disposición a fluir en el trabajo, burnout, tipología cronopsicológica, turno laboral y bienestar psicológico en una muestra multiocupacional de trabajadores venezolanos [Trabajo final de licenciatura no publicado]. Universidad Metropolitana.

Jackson, S., Ford, S., Kimiecik, J., \& Marsh, H. (1998). Psychological correlates of flow in sport. Journal of Sport and Exercise Psychology, 20(4), 358-378. https://doi.org/10.1123/jsep.20.4.358

Kakavand, A., \& Andish, S. (2016). Determining the contribution of perfectionism and perceived social support to predict the students' psychological well-being. International Journal of Humanities and Cultural Studies, Special Issue, 234243. http://www.ijhcs.com/index.php/ijhcs/article/view/636

Kanten, P., \& Yesiltas, M. (2015). The effects of positive and negative perfectionism on work engagement, psychological well-being and emotional exhaustion. Procedia Economics and Finance, 23, 1367-1375. https://doi.org/10.1016/S2212-5671(15)00522-5

Kerlinger, F., \& Lee, H. (2002). Investigación del comportamiento: métodos de investigación en ciencias sociales ( $4^{\mathrm{a}} \mathrm{ed}$.). McGraw-Hill Interamericana.

Klibert, J., Langhinrichsen-Rohling, J., \& Saito, M. (2005). Adaptive and maladaptive aspects of self-oriented versus socially prescribed perfectionism. 
Journal of College Student Development, 46(2), 141-156. https://doi.org/10.1353/csd.2005.0017

Kozier, B., Berman, A., Snyder, S., \& Erb, G. (2008). Fundamentos de enfermería: conceptos, procesos y prácticas ( $8^{\mathrm{a}}$ ed.). Pearson Educación.

Leibovich, N., \& Maglio, A. (2012). La experiencia de fluir (Flow) en médicos argentinos. IntraMed Journal, 1(2), 1-6. http://journal.intramed.net/index.php/Intramed_Journal/article/view/80

Ljubin-Golub, T., Rijavec, M., \& Jurčec, L. (2018). Flow in the academic domain: The role of perfectionism and engagement. The Asia-Pacific Education Researcher, 27(2), 99-107. https://doi.org/10.1007/s40299-018-0369-2

Marin, M., \& Bhattacharya, J. (2013). Getting into the musical zone: Trait emotional intelligence and amount of practice predict flow in pianists. Frontiers in Psychology, 4(853), 1-14. https://doi.org/10.3389/fpsyg.2013.00853

Mark, G., \& Smith, A. (2012). Occupational stress, job characteristics, coping, and the mental health of nurses. British Journal of Health Psychology, 17(3), 505521. https://doi.org/10.1111/j.2044-8287.2011.02051.x

Melrose, S. (2011). Perfectionism and depression: Vulnerabilities nurses need to understand. Nursing Research and Practice, 1-7. https://doi.org/10.1155/2011/858497

Mercado, D., \& Luna, M. (2003). Inventario Mexicano de Masculinidad y Feminidad: desarrollo psicométrico y versión preliminar. Revista Mexicana de Psicología, 20(1), 113-126.

Millán, A., \& D’Aubeterre, M. (2011). Validación de la Escala de Bienestar Psicológico en una muestra multiocupacional venezolana. Revista CES Psicología, 4(1), 52-71. http://revistas.ces.edu.co/index.php/psicologia/article/view/1255

Millán, A., \& D’Aubeterre, M. (2012). Fluir en el trabajo: un camino hacia el bienestar de los trabajadores. En M. Garassini \& C. Camilli (Eds.), La felicidad duradera: estudios sobre el bienestar en la psicología positiva (pp. 255-274). Alfa.

Millán, A., \& D’Aubeterre, M. (2013). Confirmación de la estructura factorial de la Escala de Disposición a Fluir en el Trabajo. En A. Salcedo (Ed.), Estadística en la investigación: competencia transversal en la formación universitaria (pp. 117-144). Programa de Cooperación Interfacultades, Vicerrectorado Académico, Universidad Central de Venezuela.

Millán, A., D’Aubeterre, M., \& Garassini, M. (2012, noviembre 19-23). Propiedades psicométricas de la Escala de Disposición a Fluir en el Trabajo Versión 3 (EDFT-3) [Trabajo presentado]. LXII Convención Anual de la Asociación Venezolana para el Avance de la Ciencia (AsoVAC), Caracas, Venezuela.

Millán, A., García-Álvarez, D., \& D’Aubeterre, M. (2014). Efecto de la inteligencia emocional y flujo en el trabajo sobre estresores y bienestar psicológico: análisis de ruta en docentes. Revista Colombiana de Psicología, 23(1), $207-$ 228. https://doi.org/10.15446/rcp.v23n1.37676

Morchain, B. (2011). Disposición a fluir en el trabajo como moderadora de la relación entre factores generadores de estrés y su percepción sobre el bienestar psicológico de una muestra de docentes de educación inicial del Área Metropolitana de Caracas [Trabajo final de licenciatura no publicado]. Universidad Metropolitana.

Newman, K., Maylor, U., \& Chansarkar, B. (2002). "The nurse satisfaction, service quality and nurse retention chain": Implications for management of 
recruitment and retention. Journal of Management in Medicine, 16(4-5), 271291. https://doi.org/10.1108/02689230210445095

Nilsson, K., Hertting, A., Petterson, I., \& Theorell, T. (2005, septiembre). Pride and confidence at work: Potential predictors of occupational health in a hospital setting. BMC Public Health, 5(92). https://doi.org/10.1186/1471-2458-5-92

O'Brien, S., \& Page, S. (1994). Self-efficacy, perfectionism, and stress in Canadian nurses. Canadian Journal of Nursing Research, 26(3), 49-61. https://cjnr.archive.mcgill.ca/article/view/1239

Oviedo, H., \& Campo-Arias, A. (2005). Aproximación al uso del coeficiente alfa de Cronbach. Revista Colombiana de Psiquiatría, 24(4), 572-580. https://www.redalyc.org/articulo.oa? id=80634409

Ozbilir, T., Day, A., \& Catano, V. (2015). Perfectionism at work: An investigation of adaptive and maladaptive perfectionism in the workplace among Canadian and Turkish employees. Applied Psychology, 64(1), 252-280. https://doi.org/10.1111/apps.12032

Pinto, A., \& Rodríguez, Y. (2015). La ausencia presente de madre como elemento ético-moral incongruente frente a la realidad social de un niño popular venezolano. Historia de vida de " $W$ " [Trabajo Especial de Grado de licenciatura, Universidad de Carabobo]. Repositorio Institucional UC. http://mriuc.bc.uc.edu.ve/handle/123456789/3079

Prieto, A., \& Vento, A. (2012). Validación de la versión española de la Escala Multidimensional de Perfeccionismo de Frost, Marten, Lahart y Rosenblate [Trabajo final de licenciatura no publicado]. Universidad Metropolitana.

Rhéaume, J., Freeston, M., Dugas, M., Letarte, H., \& Ladouceur, R. (1995). Perfectionism, responsibility and obsessive-compulsive symptoms. Behaviour Research and Therapy, 33(7), 785-794. https://doi.org/10.1016/00057967(95)00017-R

Sahraian, A., Fazelzade, A., Mehdizadeh, A., \& Toobaee, S. (2008). Burnout in hospital nurses: A comparison of internal, surgery, psychiatry and burns wards. International Nursing Review, 55(1), 62-67. https://doi.org/10.1111/j.1466-7657.2007.00582.x

Sánchez-Cánovas, J. (2007). Manual. Escala de Bienestar Psicológico (EBP) (2a ed.). TEA Ediciones.

Seligman, M. (2011). La vida que florece. Ediciones B.

Siblesz, M., \& Von der Heyde, A. (2013). Estilos de afrontamiento como moderador entre fuentes de estrés ocupacional y bienestar psicológico en docentes de educación básica [Trabajo final de licenciatura no publicado]. Universidad Metropolitana.

Słodkowska, J., \& Bokszczanin, A. (2012). Perfekcjonizm i stres a dobrostan psychiczny studentów uczelni wyższych. Testowanie modelu mediacyjnego. Acta Universitatis Lodziensis. Folia Psychologica, 16, 3-11. http://cejsh.icm.edu.pl/cejsh/element/bwmeta1.element.hdl_11089_4535

Stoeber, J. (2012). Perfectionism and performance. En S. Murphy (Ed.), Oxford handbook of sport and performance psychology (pp. 294-306). Oxford University Press. https://doi.org/10.1093/oxfordhb/9780199731763.013.0015

Stoeber, J., \& Damian, L. (2016). Perfectionism in employees: Work engagement, workaholism, and burnout. En F. Sirois \& D. Molnar (Eds.), Perfectionism, health, and well-being (pp. 265-283). Springer. https://doi.org/10.1007/978-3319-18582-8_12 
Stoeber, J., \& Otto, K. (2006). Positive conceptions of perfectionism: Approaches, evidence, challenges. Personality and Social Psychology Review, 10(4), 295319. https://doi.org/10.1207/s15327957pspr1004_2

Suárez, A. (2014). Justicia organizacional percibida, estilos de afrontamiento y bienestar psicológico [Trabajo final de licenciatura no publicado]. Universidad Metropolitana.

Zito, M., Colombo, L., \& Cortese, C. (2015). Il ruolo del flow at work nella professione infermieristica tra domande lavorative e disturbi psicosomatici. Giornale Italiano di Psicología, 42(3), 571-592.

https://doi.org/10.1421/81167

Received: 2018-09-18

Accepted: 2020-05-19 